\title{
Le retour au vari comme ethos de la fête (Grèce)
}

"Back to Vari" as the Ethos of Feasts in Epirus (Greece).

\section{Hélène Delaporte}

\section{(2) OpenEdition}

1 Journals

\section{Electronic version}

URL: https://journals.openedition.org/clo/1950

DOI: $10.4000 /$ clo. 1950

ISSN: 2266-1816

\section{Publisher}

INALCO

\section{Printed version}

Date of publication: 1 January 2013

ISBN: 978-2-85831-217-7

ISSN: 0396-891X

\section{Electronic reference}

Hélène Delaporte, "Le retour au vari comme ethos de la fête (Grèce)", Cahiers de littérature orale [Online], 73-74 | 2013, Online since 04 May 2015, connection on 01 July 2021. URL: http:// journals.openedition.org/clo/1950 ; DOI: https://doi.org/10.4000/clo.1950

This text was automatically generated on 1 July 2021.

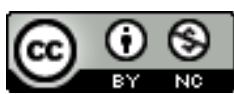

Cahiers de littérature orale est mis à disposition selon les termes de la Licence Creative Commons Attribution - Pas d'Utilisation Commerciale 4.0 International. 


\section{Le retour au vari comme ethos de la fête (Grèce)}

"Back to Vari" as the Ethos of Feasts in Epirus (Greece). Hélène Delaporte

1 À Pirsoyianni, cette année encore, la place du village est pleine. Plus de trois cents personnes ont répondu présentes pour la fête patronale, le paniyiri.Depuis que les festivités ont commencé vers huit heures du soir, tout se déroule pour le mieux. Les différents groupes familiaux se succèdent sans discontinuer sur la place pour danser ${ }^{1}$.

2 Aux alentours de minuit, alors que la fête bat son plein, Antonis, un homme d'environ soixante-cinq ans, entre en piste. Celui que beaucoup appellent l'« Américain », parce qu'il vit au Texas où il a dû s'exiler très jeune, mène à son tour la danse en cercle ouvert à laquelle prennent part les membres de sa famille. Ensemble, ils enchaînent les danses les unes aux autres comme il se doit, formant ainsi une suite d'environ un quart d'heure qui s'achève par une chanson d'amour légère au tempo enlevé. Mais ce chant à peine terminé, une lamentation instrumentale tout à fait surprenante à cet instant retentit. La fête change alors brusquement de tonalité. Les premières notes de ce miroloï (littéralement : « discours sur le destin ») ont un effet saisissant. Beaucoup de villageois s'arrêtent de converser, leurs regards convergent vers les musiciens et Antonis pour comprendre ce qui se passe.

3 C'est précisément à des instants comme celui-ci, où le rythme de la fête vacille, que le présent article entend s'attacher. Ces changements brusques attirent l'attention et provoquent, à des degrés divers, commentaires et émotions. Qu'elles interviennent à l'échelle d'une suite de danses ou à celle de l'architecture de la fête tout entière, ces ruptures de rythme relèvent toutes, semble-t-il, d'une même logique à l'œuvre : opérer un retour au caractère vari (lourd, pesant, grave) du répertoire.

4 Par l'analyse de l'intrication des différents paramètres entrant en jeu dans ces ruptures de rythme ainsi que des effets qu'elles produisent, j'émettrai l'hypothèse qu'elles constituent un trait central de l'esthétique épirote et, de ce fait, une clé essentielle à la compréhension de l'ethos de la fête. 


\section{Émigration et fête : rupture du rythme social} de la région. Ainsi, sous l'Empire ottoman et jusqu'à la fin du XIX ${ }^{\mathrm{e}}$ siècle, la vie sociale était exclusivement structurée autour d'une émigration saisonnière des hommes. Spécialisés dans la taille de la pierre et la maçonnerie, ils étaient employés dans l'ensemble de la Grèce ainsi qu'en Albanie, en Serbie, puis dans la région du Caucase et même jusqu'en Afrique (Égypte, Soudan) et au Moyen-Orient (Iran)². Selon la distance, l'absence des hommes du village était plus ou moins longue, allant de quelques mois par an à quelques années pour les destinations les plus lointaines.

$7 \mathrm{Au}$ cours $\mathrm{du} \mathrm{xx}^{\mathrm{e}}$ siècle, la pression économique fut telle, qu'à cette émigration saisonnière ancienne s'ajouta une émigration définitive qui, par vagues successives, finit par supplanter la première et assécher littéralement la terre de ses habitants.

Ces départs se firent au profit des grandes villes de Grèce (Athènes, Missolongui, Volos, Thessalonique), mais aussi, massivement, de destinations transocéaniques comme les États-Unis, l'Australie, le Canada ou bien encore européennes, essentiellement l'Allemagne et la Belgique. Enfin, de façon bien plus anecdotique, certains Épirotes partirent pour l'Égypte, le Soudan, le Congo ou l'Éthiopie.

9 Dans ces villages frontaliers de l'Albanie, on ne peut qu'être frappé par la disproportion entre le nombre de maisons et celui des résidents permanents. Pour bien saisir la situation, il faut se figurer un village typique du nord de l'Épire ainsi : cent maisons dont seulement cinq à dix sont occupées tout au long de l'année.

La vie sociale fonctionne au ralenti, car excepté quelques actifs, artisans, éleveurs ou employés, les villageois sont essentiellement des Épirotes rentrés d'exil à l'âge de la retraite. Cette situation démographique préoccupante pourrait laisser croire que les villages épirotes sont à l'abandon. Paradoxalement, il n'en n'est rien: malgré l'éloignement, les liens avec le village natal restent très étroits et, depuis leur lieu d'exil, beaucoup d'Épirotes s'impliquent dans des associations afin de récolter des fonds nécessaires à la réalisation d'infrastructures ou de travaux d'entretien ${ }^{3,}$ comme la construction d'une salle des fêtes, d'une fontaine ou encore la rénovation d'une chapelle.

11 Dans ce contexte très particulier, Noël et plus encore Pâques sont des fêtes qui voient un certain nombre d'émigrés revenir au village. C'est cependant la fête patronale (paniyiri), en été, qui constitue l'occasion de réunir l'ensemble de la communauté grâce au retour de la majorité des émigrés.

12 Par la soudaine intensification des relations qu'elle provoque au sein des villages épirotes, la fête patronale (paniyiri) constitue une véritable rupture du rythme social. 


\section{Physionomie d'une fête patronale}

13 Sans pouvoir entrer dans une description détaillée d'un paniyiri, en donner la physionomie générale apparaît toutefois nécessaire. En effet, pour schématique qu'elle soit, elle n'en donne pas moins le cadre dans lequel s'inscrivent les ruptures de rythme qui nous intéressent ici.

Lorsque tout fonctionne bien, l'on peut dire qu'entre son commencement vers huit heures du soir et son achèvement à l'aube, la fête passe par quatre phases ${ }^{4}$ :

La première est celle de l'ouverture du paniyiri. Sur la place du village ont été disposées des tables et des chaises de manière à accueillir l'ensemble des villageois et à laisser un grand espace destiné à la danse. Les musiciens - instrumentistes professionnels roms se tiennent le plus souvent sur une petite estrade. Leur ensemble (kompania) comprend un ou deux clarinettistes, un violoniste, un luthiste, un accordéoniste et un joueur de defi (tambour sur cadre) ${ }^{5}$. Alors que les gens se rassemblent progressivement et s'installent aux tables pour le début du repas, les musiciens ouvrent systématiquement le paniyiri par quelques lamentations funèbres instrumentales non mesurées ${ }^{6}$ appelées miroloyia (littéralement : « discours sur le destin »), suivies sans interruption du son par une série de chants de table, épitrapezia (littéralement : « autour de la table »), évoquant l'exil ou la mort sur des pulsations lentes. Il s'agit, comme me le dit Yorgos, un clarinettiste, de rassembler les villageois. Par l'évocation des absents, c'est l'ensemble de la communauté qui est convoquée, y compris les défunts et les exilés (Delaporte, 2011).

16 Après plusieurs suites composées de lamentations instrumentales et de chants de table, les danses peuvent commencer et la fête entrer dans sa seconde phase. Si, jusqu'ici, les musiciens prenaient l'initiative du répertoire, dorénavant ils doivent répondre aux désirs des danseurs successifs et sont rémunérés en conséquence, morceau après morceau.

17 Les premiers à occuper la piste sont les notables ou responsables des différentes associations qui se sont occupées de l'organisation du paniyiri. L'atmosphère est assez protocolaire, les danses sont lentes. Tout comme les chants de table précédents, la plupart d'entre elles évoquent les absents. En réalité, ces danses sont en tous points identiques aux chants de table. Ce sont strictement les mêmes pièces, mais cette fois-ci dansées. Ici, c'est le contexte, et notamment l'engagement du corps, qui fait catégorie et non pas les caractéristiques musicales et textuelles intrinsèques d'un morceau (Delaporte, 2010, 203).

La troisième phase, la plus longue, voit l'ensemble des familles se succéder sur la place pour danser, puis se mélanger en fonction d'affinités réelles ou relevant davantage de rapports clientélistes. L'alcool et la fatigue faisant leur œuvre, l'ambiance est plus détendue. Aux danses lentes et empreintes de gravité s'ajoute tout le reste du répertoire dansé en Épire. Les rythmes se diversifient et les tempos rapides apparaissent.

19 Vers le milieu de la nuit, la place du village est plus clairsemée et la fête entre alors dans sa quatrième phase. Les demandes des villageois alternent entre danses et chants de table, puis, peu avant l'aube, entre chants de table et lamentations instrumentales. Ainsi, du point de vue du répertoire, le paniyiri s'achève comme il avait débuté. 
Certains villages organisent dès le lendemain matin une fête de jour (imérissio paniyiri) hors du village, à proximité d'une chapelle dédiée au saint patron. Tous se retrouvent pour assister à une messe, puis les groupes familiaux s'installent pour pique-niquer. Les musiciens jouent alors pour chaque famille successivement, en commençant par des miroloyia, puis en jouant des chants de table et, dans une moindre mesure, des danses.

\section{Une esthétique de la rupture}

21 Après ce cadre général, il faut entrer dans la chair de la fête et interroger l'organisation des danses elles-mêmes. C'est à cette échelle, en effet, que se situe le cœur de la rupture de rythme.

Les danses se font en un cercle ouvert qui, mené par un premier danseur (protokhoreftis) tourne dans le sens inverse des aiguilles d'une montre. Ce protokhoreftis est considéré comme étant véritablement le seul danseur, les autres membres du cercle étant là pour l'honorer et le soutenir. C'est donc à lui que revient le droit de choisir les morceaux qu'il souhaite être joués et cela contre une rétribution aux musiciens.

Il compose ainsi une ou deux suites de danses (enotita) qui durent entre dix et quinze minutes et comportent en moyenne quatre à cinq morceaux ${ }^{7}$.

24 Tenant compte des indications verbales ou gestuelles du premier danseur, les instrumentistes passent d'un rythme à un autre, d'une chanson à une autre, le plus souvent sans interruption du son. Le premier danseur signale un changement au clarinettiste, chef de la kompania, en chantant le premier vers d'une chanson, en réclamant un type de danse, en faisant un geste de la main pour lui signifier qu'il faut passer à une autre danse et progresser dans la suite ${ }^{8}$, ou bien encore en lui indiquant un tempo. Par exemple, alors que le cercle des danseurs évolue sur un zaghorissio (danse en cinq temps typique de l'Épire, venant des villages du Zaghori), le protokhoreftis, avec autorité, intime l'ordre au clarinettiste de passer à une danse rapide et légère. Il lance : grigora! («vite!»), et accompagne son mot d'un geste de la main qui mime l'accélération. Immédiatement, les musiciens s'exécutent, et la suite se poursuit sur un tempo bien plus vif et sur un nouveau pas de base.

Véritable chef d'orchestre, le protokhoreftis dirige les musiciens afin d'élaborer la suite qu'il souhaite. Peut-il pour autant faire ce qu'il veut? Y a-t-il, au contraire, des passages obligés ou une succession précise de type de danses? À ces questions, voici ce qui me fut répondu par des musiciens : « Non, ça dépend de ce que veut le danseur, il te dit quoi jouer" (Oxhi, analogo me avto pou théli o khoreftis, avtos sou lei ti na péxis) ou " chacun fait comme il veut » (o kathénas kani opos théli). Les villageois que j'interrogeais à ce sujet ne me dirent pas autre chose, à l'instar d'Antonia: "Je danse les chansons que je veux, moi. Moi, chaque année, je danse Ta kagguélia " (khorevo ta traghoudia pou thélo 'go. Ego kathé kronia xhorevo Ta kagguélia).

Comme souvent, la distorsion est grande entre ce qui est affirmé et la réalité des pratiques. Si la décision revient au protokhoreftis, il ne peut pour autant bâtir sa suite sans respecter certaines règles. L'observation d'un grand nombre de fêtes permet de déceler des régularités dans l'élaboration des suites. Afin de vérifier ces intuitions, j'ai proposé à des musiciens des successions de danses jamais observées, à l'opposé du schéma directeur décelé : "La fête commence et je danse Olga, très vite (Olga est une danse légère qui clôt couramment une suite) et je termine par un poghonissio (danse de 
la région de Poghoni, lente et grave par laquelle les danseurs commencent très souvent) ». La réaction tout à la fois effrayée et unanime de mes interlocuteurs était sans équivoque : «Impossible, on va dire celle-là, elle n'y connaît rien! D'où elle sort ? Elle est pas d'ici!» (Dhen bori! Tha poumé avti dhen xéri! Apo pou iné avti? Apo 'dho dhen iné!)

Ainsi, sans entrer dans les détails, nous pouvons donner un profil-type de la suite de danses en Épire; il s'agit là d'une orientation générale à nuancer en fonction du déroulement de la fête. Chaque suite doit être comprise et étudiée dans le contexte précis où elle a lieu, en tenant compte de ce qui la précède et la suit bien évidemment. Il n'en reste pas moins que généralement une suite commence par des danses typiques de l'Épire, au caractère grave et au tempo lent (poghonissio, zaghorissio) dont les textes évoquent l'absence, l'exil voire la mort, puis se poursuit par d'autres danses épirotes (stamiko, sta dhio), et se termine très souvent par des danses au texte léger et au tempo rapide couramment empruntées aux régions voisines ou aux îles (nissiotika).

Il ne s'agit pas ici d'un schéma strict, mais de règles implicites indiquant un mouvement général. Les danses s'enchaînent donc suivant un continuum qui va du lent au plus rapide, de la gravité des textes à la légèreté, de danses considérées comme typiquement épirotes à une certaine ouverture aux danses panhelléniques.

Cependant, à l'intérieur de ce schéma directeur, il arrive que certains opèrent une rupture de ce continuum ascendant, allant du lent au rapide, par un retour à une danse lente et grave comme au début de la suite. À ma connaissance, il s'agit là d'un trait esthétique tout à fait propre à l'Épire. Il ne se retrouve pas ailleurs en Grèce ni dans les pays balkaniques voisins, où le schéma ascendant du lent au rapide prévaut généralement.

Je citerai l'exemple de cet épisode qui s'est déroulé en août 2008 lors du paniyiri d'Aghia Varvara, village singulier tant il est touché par l'exil'.

Venus de New York, un homme et son épouse évoluaient alternativement en première position. À la demande du mari, ils dansèrent notamment sur un rythme binaire et un tempo rapide, une chanson d'amour :

\begin{tabular}{|c|c|}
\hline $\begin{array}{l}\Delta \eta \mu \eta ́ \tau \rho \eta \mu o v \\
\Delta \eta \mu \eta ́ \tau \rho \eta \mu o v\end{array}$ & Dimitri, mon Dimitri \\
\hline $\begin{array}{l}\lambda \varepsilon \beta \varepsilon ́ v \tau \eta \mu o v \text { кl } \\
\alpha \sigma u ́ \rho \tau \eta \mu o v .\end{array}$ & Mon seigneur exaltant \\
\hline $\begin{array}{l}\Delta \eta \mu \eta ́ \tau \rho \eta, \\
\Delta \eta \mu \eta \tau \rho \alpha ́ k \eta \mu o v\end{array}$ & Dimitri, mon Dimitri \\
\hline 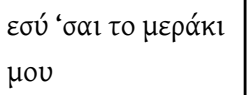 & Toi tu es mon méraki ${ }^{10}$ \\
\hline 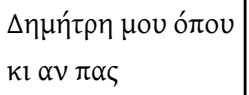 & Mon Dimitri où que tu ailles \\
\hline$[\ldots] \mu \eta \mu \varepsilon \xi_{\varepsilon \chi \chi \alpha ́} \varsigma$ & {$[\ldots]^{11}$ ne m'oublie pas } \\
\hline 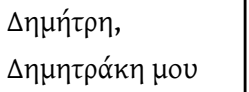 & Dimitri, mon Dimitri \\
\hline
\end{tabular}




\begin{tabular}{|c|c|}
\hline 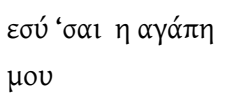 & Toi tu es mon amour \\
\hline 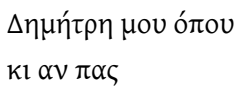 & Mon Dimitri où que tu ailles \\
\hline$[\ldots] \mu \eta \mu \varepsilon \xi_{\varepsilon \chi \nu \alpha ́}$ & {$[\ldots]$ ne m'oublie pas } \\
\hline
\end{tabular}

Les musiciens prolongèrent cette danse par un air de Thrace sur le même rythme, toujours en accélérant le tempo. Alors que le cercle des danseurs s'emballait, le mari, passé en première position, lança aux musiciens : «Lent! Poghonissio!, Lent! Lent !» (Argo! Poghonissio vari! Argo!) Ceux-ci jouèrent alors un poghonissio instrumental très lent.

Ce genre de basculement peut avoir lieu aussi dans la dernière partie du paniyiri. Cela fut le cas, par exemple, le 15 août 2007 peu avant le lever du jour à Pirsoyianni. Les musiciens lui jouaient un kalamatianos (rythme panhellénique) instrumental sur un tempo rapide, lorsqu'un homme demanda soudain ce chant d'exil sur un rythme de poghonissio :

\begin{tabular}{|c|c|}
\hline $\begin{array}{l}\text { Ax } \tau \omega ́ \rho \alpha \sigma \tau \alpha \\
\xi \varepsilon \chi \omega \rho i ́ \sigma \mu \alpha \tau \alpha\end{array}$ & Ah maintenant la séparation \\
\hline 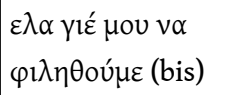 & $\begin{array}{l}\text { Viens, que nous nous } \\
\text { embrassions }\end{array}$ \\
\hline 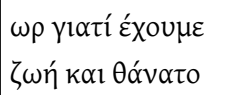 & $\begin{array}{l}\text { Parce qu'il y a la vie et la } \\
\text { mort }\end{array}$ \\
\hline 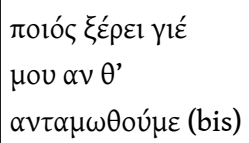 & Qui sait quand nous nous reverrons? \\
\hline 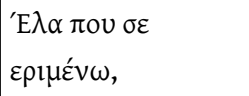 & Viens où je t'attends \\
\hline 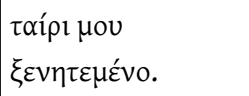 & mon époux exilé \\
\hline 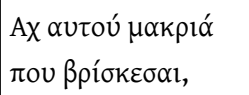 & $\begin{array}{l}\text { Là-bas, au loin où tu te } \\
\text { trouves, }\end{array}$ \\
\hline 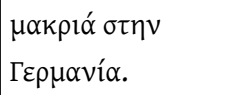 & Loin en Allemagne \\
\hline 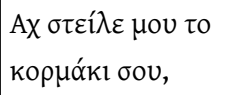 & Ah, envoie-moi ton petit corps \\
\hline 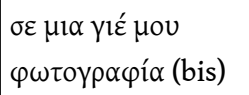 & En photographie \\
\hline 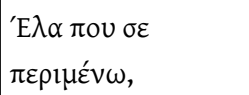 & Viens ou je t'attends, \\
\hline 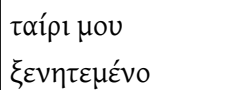 & mon époux exilé \\
\hline
\end{tabular}



musiciens passent d'un rythme en $2 / 4$ à un rythme en $4 / 4$ dans un cas et d'un rythme en $7 / 4$ à un rythme en $4 / 4$ dans l'autre. Le tempo est très fortement ralenti : deux fois plus lent. Les musiciens passent d'une mélodie d'ossature diatonique ${ }^{12}$ à une mélodie d'ossature pentatonique anhémitonique ${ }^{13}$. De plus, le registre change. Les clarinettistes, qui jouaient dans le registre aigu ou suraigu de leur instrument, changent subitement pour le registre grave. Enfin, que les danses soient instrumentales ou jouées dans leur version chantée, il est clair que l'on passe d'une certaine légèreté à la gravité. Cette dernière a ici une double sémantique, à la fois musicale et textuelle. Cette rupture de rythme attire l'attention des autres villageois. Sans être forcément commentée, on voit bien qu'elle est remarquée. Comme, par exemple, ces deux hommes, en grande conversation, qui, alertés par ce basculement sonore, scrutent un instant la piste de danse et notent qui en est l'acteur. Le recours à ce code esthétique n'est donc pas systématique, mais s'il est employé à bon escient, il peut engendrer des commentaires positifs, par exemple : « Celui-là, il sait » (avtos xéri), ou : « Il est d'ici, celui-là » (Apo dho 'né avtos), ou bien encore « Pallicari! Comme son père! " (Pallicar! San o patéras tou).

obtenir cette rupture de rythme, les Épirotes s'adressent aux musiciens de plusieurs manières. Soit ils disent le premier vers de la chanson qu'ils souhaitent: «Parce que les montagnes sont noires" (Yiat' iné mavra ta vouna) - on notera que, très souvent, le morceau est joué par la suite dans sa version instrumentale; soit ils indiquent un rythme de danse et le caractère qui lui est associé ; c'est le cas lorsque l'un d'entre eux enjoint de jouer "un poghonissio lourd " (éna poghonissio vari). Ou bien, ce qui est très courant, les danseurs n'indiquent ni texte, ni rythme particulier, mais un trait esthétique : «lent!» (argo) ou encore « lourd, grave » (vari). Ces termes en euxmêmes suffisent aux musiciens pour savoir ce qu'ils doivent jouer, quels rythmes de danse (poghonissio ou zaghorissio) choisir et quelle interprétation est attendue d'eux.

36

pu remarquer que c'est toujours le caractère lourd ou grave qui est exigé avec insistance quand on s'adresse aux musiciens. Si les instrumentistes ne répondent pas suffisamment à cette injonction, les danseurs insistent assez vertement. « Lourd ! Je t'ai dit! Tu n'entends pas?» (Vari! Sou ipa! Dhen akous ?) lance, courroucé, un danseur à un clarinettiste dont il estime que le jeu ne correspond pas assez au caractère voulu. Et, à la fin d'une suite, quand le danseur estime que le rythme n'est pas assez rapide à son goût, il le fait bien entendu savoir. Mais je n'ai jamais perçu autant d'agacement voire d'enragement que lorsqu'il s'agissait d'obtenir l'interprétation soignée d'une pièce grave. Dans ce domaine, l'exigence ne souffre aucun compromis.

Chaque fois que j'ai demandé à des Épirotes de caractériser leur musique - et ce, quels qu'aient été mes interlocuteurs - les adjectifs employés étaient le plus souvent : vari (« lourd, pesant »), arga (« lent »), siguenitika (« émouvant »), lipitéra (« triste»; " nos chansons sont tristes" - ta dika mas traghoudia iné lipitéra) ou bien encore ponéména (" plaintif »). Comme le dit cet Épirote : « Nous chantons en pleurant et nous pleurons en chantant" (Emis traghoudhoumé klaigondas kai klémé traghoudondas). Ou, comme le constate cet autre: "Nos chansons épirotes sont toutes lourdes, des pièces lourdes " (Ta dika mas ta ipirotika iné olo vari, varia komatia).

Ce champ lexical est celui des lamentations funèbres ou des danses graves comme les poghonissia ou zaghorissia. Il ne dit rien de la légèreté, de la gaîté ou de la grivoiserie de certaines chansons épirotes. Il est simplement symptomatique que ce répertoire plus léger ne soit jamais mis en avant quand il s'agit de définir la musique de l'Épire. Seule 
une partie du répertoire semble porter l'identité épirote. Le caractère vari y est tellement associé que lorsqu'un danseur demande une "danse à nous" (éna diko mas) ou encore "une danse de Piroyianni », du nom d'un village (éna pirsoyianitiko), il est évident que les musiciens joueront une pièce au caractère grave et lourd.

Ce terme de vari semble donc contenir et concentrer tous les autres termes cités plus haut pour décrire la musique épirote. Sa sémantique est multiple : il définit tout à la fois un rythme, une pulsation, un registre musical (grave) et textuel (exil, mort), une tension, une lourdeur, une pesanteur dans le jeu des musiciens comme dans la danse des villageois. Il évoque aussi la douleur et le poids des sentiments.

Que se joue-t-il lorsqu'un danseur rompt le schéma ascendant d'une suite de danses par le retour au caractère vari initial, sinon l'essentiel: le rappel, l'affirmation de son identité comme épirote? Dans des instants de basculement comme celui-ci, ce qui se joue c'est son «ancrage » dans la communauté pour reprendre le mot de Véronique Nahoum-Grappe au sujet de la participation de chacun aux fêtes populaires (NahoumGrappe, 2012).

\section{L'hommage à la mère ou la manipulation virtuose d'un retour au vari}

41 Cette rupture de rythme par un retour au caractère vari peut aussi être observée à l'échelle de la fête tout entière. Revenons sur l'exemple mentionné en introduction. Alors que le paniyiri du village de Pirsoyianni était largement entré dans sa troisième phase - celle qui voit les danseurs se succéder sur la place au son de l'ensemble du répertoire et les suites se finir sur des tempos enlevés - Antonis décida de prendre son tour comme protokhoreftis.

42 Âgé de soixante-cinq ans environ, Antonis vit au Texas où il posséderait deux usines de textile. Il fait partie de ces notables qui apportent leur soutien financier à différentes rénovations ou constructions d'infrastructures dans leur village natal. C'est un homme respecté, que les musiciens ont tout intérêt à contenter. Kostas et Yorgos, les clarinettistes de la kompania systématiquement souhaitée pour animer le paniyiri de Pirsoyianni, connaissent ses goûts : ils savent ce qu'ils doivent jouer pour le satisfaire, le surprendre et faire surgir chez lui l'émotion tant recherchée lors de ces fêtes. Contrairement à d'autres, Antonis ne donne que peu d'indications aux musiciens. Il saute aux yeux et aux oreilles de tous qu'il n'a pas besoin de batailler pour obtenir une bonne prestation de leur part. Et cela signe, dans son cas, la marque de son prestige.

Preuve supplémentaire de son charisme, il obtient des musiciens, jusque-là installés sur une estrade et retranchés en partie derrière leur système de sonorisation, qu'ils abandonnent leur confort de jeu et viennent au plus près des danseurs jouer en acoustique. Privilège que les musiciens n'accordent pas facilement et qui constitue à cet instant une première rupture de rythme, dans l'espace s'entend.

Ce jour-là, Antonis dansa, avec sa paréa (groupe familial et/ou groupe d'amis), une première suite, puis une seconde qui se termina, comme attendu, par une danse au tempo enlevé et au texte léger ${ }^{14}$ :

\begin{tabular}{|c|c|}
\hline 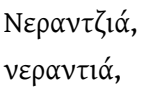 & $\begin{array}{l}\text { Mon petit oranger, } \\
\text { mon petit oranger }{ }^{15}\end{array}$ \\
\hline
\end{tabular}




\begin{tabular}{|c|c|}
\hline 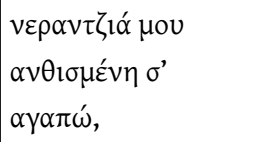 & $\begin{array}{l}\text { Mon petit oranger fleuri, } \\
\text { je t'aime }\end{array}$ \\
\hline 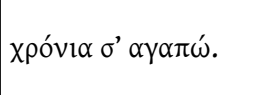 & $\begin{array}{l}\text { Depuis des années, je } \\
\text { t'aime. }\end{array}$ \\
\hline 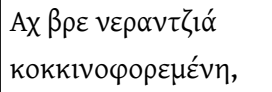 & $\begin{array}{l}\text { Ah mon petit oranger } \\
\text { de rouge vêtu }\end{array}$ \\
\hline 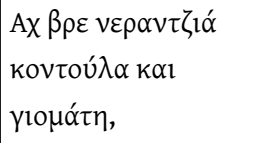 & $\begin{array}{l}\text { Ah mon oranger, petit et } \\
\text { généreux }\end{array}$ \\
\hline 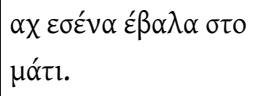 & $\begin{array}{l}\text { C'est toi qui m'as tapé } \\
\text { dans l'œil }\end{array}$ \\
\hline 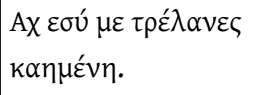 & $\begin{array}{l}\text { Tu m'as rendu fou mon } \\
\text { petit }\end{array}$ \\
\hline 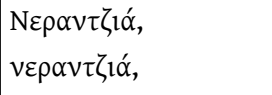 & $\begin{array}{l}\text { Mon petit oranger, mon } \\
\text { petit oranger }\end{array}$ \\
\hline 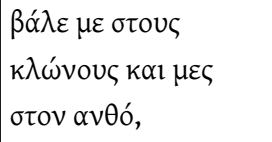 & $\begin{array}{l}\text { Mets-moi sur tes rameaux } \\
\text { et dans tes fleurs }\end{array}$ \\
\hline 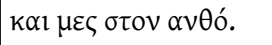 & Et dans tes fleurs \\
\hline 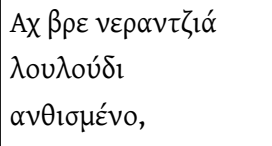 & $\begin{array}{l}\text { Ah mon petit oranger } \\
\text { plein de fleurs écloses }\end{array}$ \\
\hline 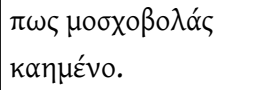 & $\begin{array}{l}\text { Comme tu embaumes, } \\
\text { mon petit }\end{array}$ \\
\hline 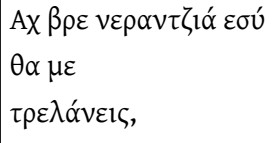 & $\begin{array}{l}\text { Ah mon petit oranger, toi } \\
\text { tu vas me rendre fou }\end{array}$ \\
\hline 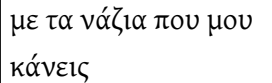 & $\begin{array}{l}\text { Avec les minauderies que } \\
\text { tu me fais }\end{array}$ \\
\hline
\end{tabular}

45 La danse terminée, les autres danseurs applaudirent Antonis et certains l'embrassèrent, marque d'affection assez peu courante. Puis, alors que la place résonnait encore des paroles de cette chanson évoquant un amoureux transi que sa belle s'amusait à faire languir, Antonis entraîna les musiciens vers sa mère très âgée, restée assise. À son intention, ils commencèrent une lamentation instrumentale (miroloi) dont les premières notes eurent un effet saisissant à ce point de la fête, alors que les paroles de la chanson légère étaient encore dans toutes les têtes.

À cet instant, en effet, beaucoup de conversations cessèrent et, de tous les coins de la place, les regards se tournèrent vers les protagonistes de la scène, chacun cherchant à comprendre les raisons de cette soudaine lamentation instrumentale. Debout comme les musiciens, Antonis lança quelques dollars en direction de sa mère. Très émue, cette femme de quatre-vingt-dix-huit ans, dissimula ses larmes derrière sa main. Sur ses 
lèvres se lisait un discret sourire. Entourée de ses proches, cette petite dame portant le deuil irradiait d'émotion contenue.

Les musiciens poursuivirent avec un chant d'exil qu'ils savaient particulièrement apprécié d'Antonis et dont la gravité contrastait avec la légèreté du chant d'amour précédent :

\begin{tabular}{|c|c|}
\hline 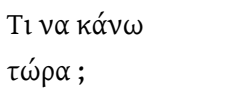 & Que vais-je faire maintenant? \\
\hline 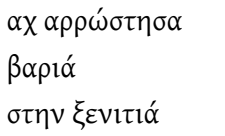 & $\begin{array}{l}\text { Ah je suis tombé gravement } \\
\text { malade à l'étranger }\end{array}$ \\
\hline 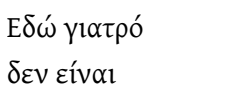 & Ici de médecin il n'y a pas \\
\hline 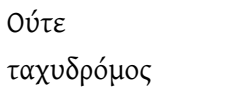 & Ni de facteur \\
\hline 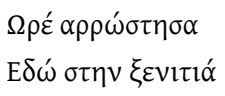 & $\begin{array}{l}\text { Ah, je suis tombé malade ici } \\
\text { à l'étranger }\end{array}$ \\
\hline 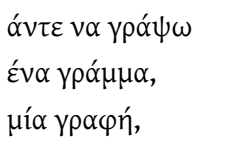 & $\begin{array}{l}\text { Allez, que j'écrive une lettre, } \\
\text { un écrit }\end{array}$ \\
\hline 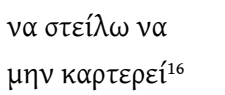 & $\begin{array}{l}\text { Que je l'envoie, qu'on } \\
\text { n'attende pas impatiemment }\end{array}$ \\
\hline 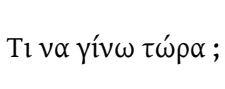 & $\begin{array}{l}\text { Que vais-je devenir } \\
\text { maintenant? }\end{array}$ \\
\hline
\end{tabular}

Ce grand fils de soixante-cinq ans caressa doucement les cheveux de sa mère, puis s'écarta pour sécher ses propres larmes dans un grand mouchoir de tissu qu'il pressa sur ses yeux. Durant le chant d'exil qui suivit la lamentation, sa mère agita en mesure sa main droite en direction des musiciens comme le font les premiers danseurs quand ils sont sur la piste. Ce geste était précieux, tant il soulignait que les mouvements lui étaient difficiles. Car lorsqu'elle ne séchait pas ses larmes, cette main venait régulièrement se reposer sur la canne où l'autre prenait déjà appui.

La musique se poursuivit ; soutenue de part et d'autre, la mère d'Antonis parvint à se lever pour danser un poghonissio lent. Après quelques pas d'une danse presque immobile, elle bascula très légèrement la tête en arrière, signifiant aux instrumentistes qu'elle souhaitait s'en arrêter là.

50 Ses proches l'aidèrent alors à se rasseoir et tous vinrent l'embrasser. Les flashs crépitaient, car beaucoup souhaitaient immortaliser ce beau moment. Après la sidération du début, l'approbation semblait dominer parmi les convives. Que s'était-il donc passé?

51 Antonis opéra ici plusieurs ruptures. La première, on l'a dit, fut une rupture dans l'espace. Les musiciens qui se trouvaient sur une estrade, vinrent jouer face au premier danseur puis acceptèrent d'aller jusqu'à la table de la mère d'Antonis. 

lamentation renvoyaient directement à l'ouverture de la fête. Au sens strict, la rupture de rythme est donc totale : Antonis demande la seule pièce du répertoire qui ne soit pas mesurée. Au milieu des danses, cette absence de pulsation régulière est saillante et comporte une grande part de transgression dans la mesure où elle bouleverse les codes habituels. Par ces quelques mesures de miroloï, Antonis rend hommage à sa mère qui porte de nombreux deuils, dont celui de son mari. Ce faisant, il personnalise la lamentation. Mais, dans ce contexte de fête sur la place du village, les miroloyia sont toujours adressés à l'ensemble de la communauté. En agissant ainsi, Antonis anticipe en quelque sorte et impose ce qui se fait habituellement le lendemain, hors du village, lorsque les musiciens jouent successivement pour chaque famille en commençant par une lamentation personnellement adressée.

En le poussant à l'extrême, Antonis utilise ce code esthétique du retour au vari de façon virtuose. La réaction qu'il provoque est alors sans équivoque. L'émotion, palpable au sein des membres de sa famille, déborde du cadre strict de sa parentèle. Beaucoup semblent touchés par cet hommage magistral et inattendu, au beau milieu de la fête, à cette mère qu'il a dû quitter pour s'exiler aux États-Unis. Au cours de ces fêtes où chaque famille est touchée par la douleur de la séparation, l'émouvante irruption de la lamentation est, dans ces conditions, bien perçue.

Même si elle est transgressive, cette rupture du rythme de la fête semble conférer à Antonis un rôle fédérateur. Par l'émotion qu'il a suscitée, il contribue à créer un lien au niveau de la communauté villageoise. Lorsque, bien après la fête, je visionnais la séquence vidéo de cette scène chez un des clarinettistes, sa femme, très émue, me dit : «C'est un Monsieur, celui-là ! » (Kirios avtos!)

\section{Conclusion}

L'exemple d'Antonis nous montre de manière paradoxale que celui qui sait manipuler à bon escient les ruptures de rythme et de tempo apparait comme le garant de l'esthétique et des valeurs épirotes. En jouant avec les normes, Antonis manifeste justement une grande maîtrise de celles-ci. Ainsi, que ce soit à l'échelle d'une suite de danses ou de l'architecture de la fête tout entière, une même logique - utilisée avec plus ou moins de brio ou d'éclat - est en définitive à l'œuvre. Il s'agit d'opérer un recentrage vers ce que les Épirotes estiment être le caractère essentiel de leurs chants et de leur musique : le caractère vari.

Comme nous l'avons vu, cet adjectif que l'on peut traduire par lourd, pesant et grave caractérise tout à la fois un sentiment, un état d'esprit, un registre textuel, mais aussi musical, des rythmes spécifiques, un tempo lent et une échelle musicale pentatonique. Mais ce qui semble définir de manière profonde la fête en Épire, ce n'est pas seulement cette gravité polysémique du répertoire, mais plus encore une certaine forme de rupture de rythme qui opère un retour à la gravité.

58 Le danseur, en faisant surgir de la gravité au sein de la légèreté, rend honneur aux ancêtres, aux absents, défunts ou exilés. Cet hommage est rendu effectif, non pas 
seulement par l'emploi d'un répertoire spécifique, mais surtout par l'utilisation de celui-ci dans une dynamique. L'efficacité vient du mouvement de retour au vari. Tout comme les Épirotes reviennent de leur lieu d'exil vers leur village natal ou celui de leurs ancêtres pour assister à la fête, ils ne cessent pendant celle-ci d'effectuer un mouvement de renvoi au répertoire le plus grave, celui qui semble seul porter l'identité épirote. Le retour au vari signe l'ethos de la fête en Épire. Tout se passe comme si la fête n'était que tension vers la gravité avec de nécessaires échappatoires vers plus de légèreté. Habités par la douleur du retour (nostalgie), les Épirotes n'en finissent pas de revenir à la gravité. Ils se retrouvent pour chanter l'absence, la séparation, l'impossibilité d'être ensemble.

\section{BIBLIOGRAPHY}

CARAVELLI, Anna, 1985, The Symbolic Village: Community Born in Performance, Journal of American Folklore, 98, pp. 259-286.

DAMIANAKOS, Stathis, 1996, le Paysan grec : défis et adaptations face à la société moderne, Paris, L'Harmattan.

DELAPORTE, Hélène, 2006, Quand les chanteurs sont grecs et les musiciens tsiganes : la musique traditionnelle en Épire, Études balkaniques, 13, p. 281-295.

DELAPORTE, Hélène, 2010, De l'enterrement à la fête. Parcours d'un texte funèbre en Épire, Grèce, in Claude Calame, Florence Dupont, Bernard Lortat-Jacob et Maria Manca (dir.), la Voix actée : pour une nouvelle ethnopoétique,Paris, Kimé, p. 195-210.

DELAPORTE, Hélène, 2011, l'Ambiguïté des discours, Cahiers de littérature orale, $\mathrm{n}^{\circ}$ 69, p. 34-55. FUSULIER, Bernard, 2011, le Concept d'ethos, Recherches sociologiques et anthropologiques [En ligne], 42-1, consulté le 8 avril 2015.

LAMBERT, Jean, 2004, Temps musical et temps social au Yémen : la suite musicale dans le magyal de Sanaa, l'Homme, « Musique et anthropologie », $\mathrm{n}^{\circ}$ 171-172, p. 151-172.

LORTAT-JACOB, Bernard, 1980, Musique et fêtes au Haut-Atlas, Paris, Ehess/Mouton et Société française de musicologie. Avec $1 \mathrm{CD}$-audio encarté.

NAHOUM-GRAPPE, Véronique, 2012, Temps de la fête, temps de l'ivresse, anthropologie des rythmes sociaux, Rhuthmos, 10 février 2012, consulté le 8 avril 2015.

PANOPOULOS, Panayotis, 2005, Retour au village natal, Ethnologie française 2, vol. 35, p. 243-253.

\section{NOTES}

1. Je remercie Sandra Bornand, Maria Manca, Bernard Lortat-Jacob, Anne-Florence Borneuf, Daphné Stergiopoulos, Éric Pichon et les correcteurs anonymes des CLo pour leurs nombreuses remarques et suggestions. 
2. Pour plus de détails, voir DAmianaKos $(1996,98)$.

3. Ce paradoxe a été très bien décrit par DAMIANAKos (1996, 91-119).

4. Il s'agit ici de donner un mouvement général et non pas un déroulement strict de la fête.

5. Pour davantage d'éléments concernant les musiciens traditionnels d'Épire, on pourra consulter DELAPORTE (2006).

6. Sans pulsation régulière. Les miroloyia sont les seules pièces du répertoire épirote à être ainsi entièrement non mesurées.

7. Ce chiffre est une moyenne car le nombre de danses et leur durée ne sont pas fixes. Il dépend de paramètres liés aux enjeux de la fête et notamment des rapports de force instaurés entre danseurs et musiciens.

8. Le clarinettiste est chargé de faire une proposition qui aille dans le sens des désirs du danseur ; si ce n'est pas le cas, il sera immédiatement recadré.

9. La cinquantaine de maisons modestes aux potagers soignés et d'autres aux jardins hirsutes, les trois belles fontaines aux abords de la place, le café, le bon état des routes et des maisons, la population animée et joyeuse, rien de tout cela ne permet de deviner qu'en dehors des quelques jours qui entourent la fête, le village est vide. L'hiver, seul un berger habite ces lieux. Au milieu de ce brouhaha, rien n'indique que tous, sauf un, ont fait cent, deux cents, huit cents ou dix mille kilomètres pour être présents ce jour-là.

10. To méraki : mot polysémique signifiant envie, désir, regret, nostalgie et entrain.

11. Mot inaudible.

12. Mélodie basée sur une échelle contenant sept degrés, composée de cinq tons et de deux demi-tons. Par exemple, du grave vers l'aigu : do, ré, mi, fa, sol, la, si.

13. Mélodie basée sur une échelle comprenant cinq degrés dans l'octave sans intervalle de demiton. Par exemple, sol, la, do, ré, mi.

14. Dans cet enregistrement, ne figure que la fin de la chanson, autrement dit, les dernières mesures instrumentales. Il s'agit d'une chanson dont tous ont les paroles en tête, car elle est extrêmement demandée et chantée durant les fêtes. Voilà pourquoi il m'a semblé possible d'en commenter le texte ici bien que l'enregistrement vidéo ne permette pas de l'entendre.

15. Il s'agit d'une variété d'oranger amer qui produit beaucoup de fleurs et de fruits.

16. ti na kano tora / ti na yino tora / axh arhostissa varia sti xénitia / Edho yiatros dhen iné/Oute taxhidromos / oré arhostissa edho stin xénitia / Andé na grapso éna gramma mia grafi / na steilo na min kartéri.

\section{ABSTRACTS}

In Epirus, a region of north-west Greece strongly affected by emigration, patron saint's feasts (paniyiria) are the opportunity for the entire village community to gather. From abroad or major cities of Greece, Epirots come back to their home villages for the occasion.

This article examines the rhythm breaks that can be observed in dance suites as well as during the feast as whole. The article shows that Epirots regularly keep coming back to the heavyest repertoire (vari), the one which, according to them, carries the Epirot identity. "Back to vari" is the ethos of feasts in Epirus.

En Épire, région du nord-ouest de la Grèce très fortement touchée par l'émigration, les fêtes patronales (paniyiria) voient l'ensemble de la communauté villageoise se rassembler. Depuis 
l'étranger ou les grandes villes de Grèce, les Épirotes retournent dans leur village d'origine pour cette occasion.

Cet article étudie les ruptures de rythme que l'on peut observer à l'échelle d'une suite de danses comme de la fête tout entière. Il montre que les Épirotes ne cessent au cours de celle-ci de revenir au répertoire le plus grave (vari), précisément celui qui selon eux porte l'identité épirote. Le retour au vari signe l'ethos de la fête en Épire.

\section{INDEX}

Geographical index: Épire, Grèce

Subjects: anthropologie (Europe), ethnomusicologie

Keywords: Epirus, Musical Aesthetic, Ethos, Memory, Feast, Ancestor, Rhythm

Mots-clés: ancêtre, esthétique musicale, ethos, mémoire, fête, rythme

\section{AUTHOR}

\section{HÉLĖNE DELAPORTE}

Centre de recherche en ethnomusicologie (Crem) 